\title{
Analysis and Simulation of Digital Baseband Transmission System Basing on SIMULINK
}

\author{
Geng Wang ${ }^{1, a}$, Wei Cheng ${ }^{1, b}$ \\ ${ }^{1}$ The School of Electronic and Information Engineering, Hubei University of Science and \\ Technology,Xianning, 437100,China \\ awanggeng20001982@126.com, ${ }^{\text {b7}} 799040609 @ q q . c o m$
}

Keywords: SIMULINK; digital baseband transmission system;eye diagram;intersymbol interference

\begin{abstract}
Based on the analysis of digital baseband system transmission characteristics, SIMULINK software is used to simulate the digital baseband transmission system. Having been fully considered the possible factors affecting the baseband signal in the transmission process, a basic model of digital baseband transmission system is established, a system model which can simulate and realize the function of digital baseband signal transmission. The influence of channel noise on system performance is analyzed by eye diagram and bit error rate. It shows that the larger the channel SNR is, the smaller the bit error rate is, and the better the transmission quality is, which is consistent with the theoretical analysis .From the simulation results and bit error rate, the digital baseband transmission system model meets the basic design requirements.
\end{abstract}

\section{Introduction}

The traditional principle of communication experiments are usually carried out using the hardware experimental box. In the actual teaching, this hardware experimental box there are some shortcomings: analysis tools only oscilloscope, can only be a simple signal waveform observation, can not signal spectrum analysis[1]; equipment fixed in the experimental box; students only through the experimental box Students can not modify the equipment parameters, resulting in many experimental phenomena observed;) Experimental time and place fixed, students can not schedule according to their own learning time of the experiment. These problems restrict the realization of the teaching objectives.

In this paper, MATLAB / SIMULINK simulation software is introduced to the traditional teaching. Using the powerful simulation function of MATLAB / SIMULINK software, the design and simulation analysis of the important digital baseband transmission system[2] in the course of communication theory is taken as an example. To deepen students' understanding of related technology, the results in the form of graphic images intuitive to show, so that students from the tedious calculation of the freed, the focus on the concept, principles and methods of understanding, to mobilize the enthusiasm of students, Deepen students' understanding of teaching contents, and receive good teaching results.

\section{Analysis of Digital Baseband Transmission System Model}

Baseband signal, refers to the message source message directly through the converter into electrical signals. In the data communication process, do not use the carrier modulation and demodulation device to transmit baseband signal directly to the system, called the baseband transmission system[3]. It is not only used for low-speed data transmission, Transmission, but also for high-speed data transmission; Finally, theoretically can prove that any use of linear modulation of the band transmission system, can always be an equivalent baseband transmission system replaced. In the baseband transmission system, a series of baseband signal waveforms are converted into corresponding transmission baseband waveforms, and then sent to the channel. The signal is transmitted through the channel.On the one hand, the signal is distorted by the influence of the channel characteristic,the signal is superimposed by the additive noise in the channel, resulting in 
random distortion of the signal[4]. As a result, the baseband signal arriving at the receiving side has been distorted. To this end, the receiver must first arrange a receiver filter, so that as much as possible to suppress noise, leaving the signal smoothly. However, in the output signal of the receive filter, there is always distortion and noisy mixing. Therefore, in order to improve the reliability of the receiving system, usually at the output of the receiving filter to arrange an identification circuit, the identification circuit is commonly used sampling decider, it is in each receive baseband waveform near the center of the signal sampling, The sample value is then compared to the decision threshold. If the sampling value is greater than the threshold value, it is judged as "high" level, otherwise it is judged as "zero" level. This results in a series of new baseband signals regenerating the baseband waveform. According to the above signal transmission process, a baseband transmission system can be summarized by the model shown in Fig1:

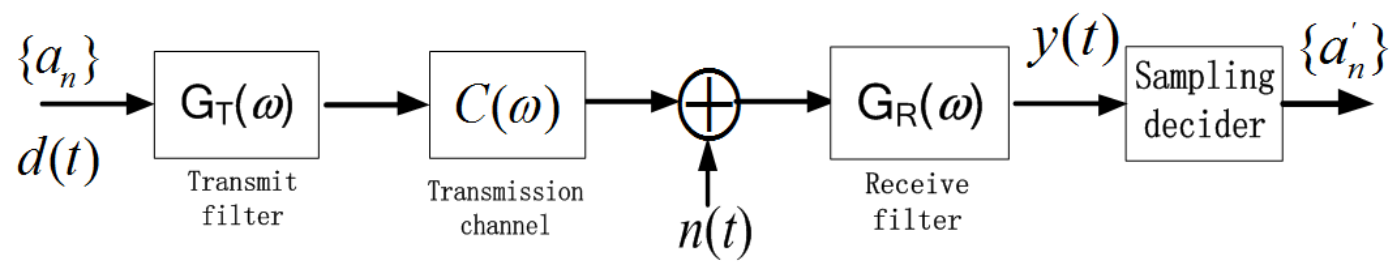

Fig.1 Baseband Transmission System Model

$a_{n}$ is the transmitted input signal, in the binary case, an corresponds to the value of 0,1 (or $-1,1$ ), the binary baseband signal $\mathrm{d}(\mathrm{t})$ time domain is expressed as:

$$
d(t)=\sum_{-\infty}^{\infty} a_{m} \delta\left(t-m T_{b}\right)
$$

The signal s ( $\mathrm{t})$ generated by the transmit filter is:

$$
s(t)=\sum_{-\infty}^{+\infty} a_{m} g_{T}\left(t-m T_{b}\right)
$$

The output signal $r(t)$ of the receiving filter is:

$$
r(t)=\sum_{-\infty}^{+\infty} a_{m} g_{R}\left(t-m T_{b}\right)+n(t)
$$

Where $\mathrm{n}(\mathrm{t})$ is the noise in the transmission channel.

$$
\begin{aligned}
& g_{R}\left(t-m T_{b}\right)=\left\{\begin{array}{l}
g_{1}\left(t-m T_{b}\right), " 1 " \\
g_{2}\left(t-m T_{b}\right), " 0 "
\end{array}\right. \\
& g_{R}(t)=\frac{1}{2 \pi} \int_{-\infty}^{+\infty} G_{T}(\omega) C(\omega) G_{R}(\omega) e^{j \omega t} d \omega
\end{aligned}
$$

$g_{R}(t)$ depends on the transfer function of the baseband network formed by the signal passing through the transmission filter, the transmission signal, the reception filter.

$$
H(\omega)=G_{T}(\omega) C(\omega) G_{R}(\omega)
$$

For the base-band transmission system without intersymbol interference, the Nyquist criterion should be satisfied. However, considering the actual filter implementation and timing requirements, the base-band transmission system usually adopts the raised-cosine spectrum characteristic. The transfer function is:

$$
H(\omega)= \begin{cases}T_{b} & 0 \leq|\omega|<\frac{(1-\alpha) \pi}{T_{b}} \\ \frac{T_{b}}{2}\left[1+\sin \frac{T_{b}}{2 \alpha}\left(\frac{\pi}{T_{b}}-\omega\right)\right] & \frac{(1-\alpha) \pi}{T_{b}} \leq|\omega|<\frac{(1+\alpha) \pi}{T_{b}} \\ 0 & |\omega| \geq \frac{(1+\alpha) \pi}{T_{b}}\end{cases}
$$

The corresponding impulse response function $\mathrm{h}(\mathrm{t})$ is:

$$
h(t)=\frac{\sin \pi t / T_{b}}{\pi t / T_{b}} \cdot \frac{\cos \alpha \pi t / T_{b}}{1-4 \alpha^{2} t^{2} / T_{b}{ }^{2}}
$$




\section{Simulation of Digital Baseband Transmission System}

The baseband transmission model is established. The transmitted data is a binary bipolar non-return-to-zero code, the transmit filter is a square root raised cosine filter, the roll-off coefficient is 0.5, the channel is an additive Gaussian channel, and the receive filter matches the transmit filter . The transmit data rate is $1000 \mathrm{bps}$, and the eye diagram is displayed by the eye diagram display module in the communication module library. In the eye diagram [5]display module need to set: (1) each data sampling point, set to 10. (2) The number of symbols displayed per scan can be set to 2, so that the eye diagram will show 2 symbol time widths. (3) Displays the number of sweep waveform tracks that are retained. Default values can be used. (4) The default value can also be used for the number of new tracks to be displayed at a time. (5) Discrete-Time Eye Diagram Scope module can simultaneously display the same phase and orthogonal waveform eye on the road[6].

Design system simulation sampling rate of 1e4HZ[7], filter sampling rate is equal to the system simulation sampling rate. Digital signal rate of $1000 \mathrm{bps}$, so before entering the transmit filter requires 10 times the rate of rise and fall, after receiving and decoding rate of 10 times to restore the signal transmission bit rate. Simulation model shown in Fig2, which the system is divided into binary

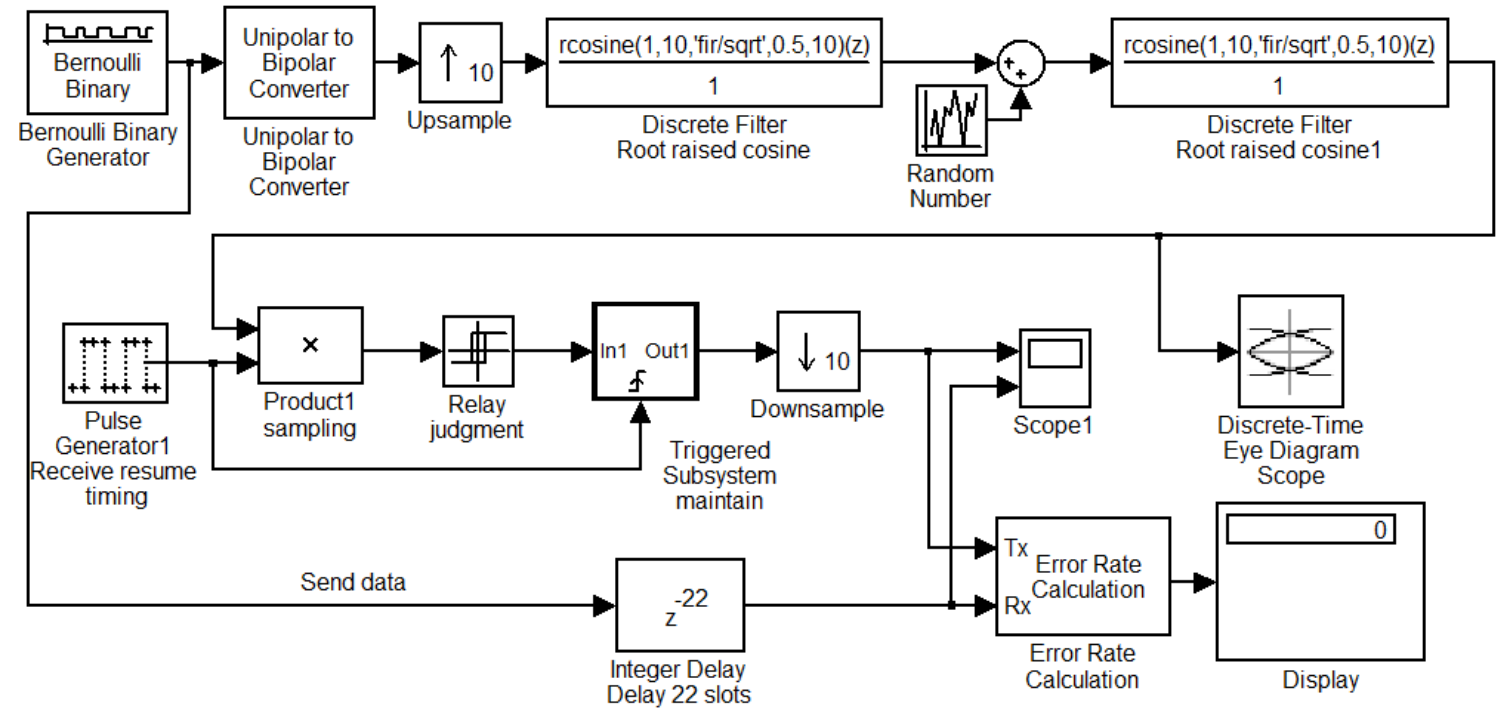

Fig.2 Simulation Test Model

source, the transmit filter, Gaussian channel, receive the matched filter, receive sampling, decision recovery and signal measurement 7 parts. The binary source outputs a bipolar non-return-to-zero code and provides raw data to the receiver for comparison and statistical error rates. The transmit and receive filters are matched to each other and are square root raised cosine filters. The Gaussian channel is implemented using a simple random number generator and adder. Since the reception timing is assumed to be ideal, a rectangular pulse of $1000 \mathrm{~Hz}$ can be realized as a recovery timing pulse by a pulse generator, and a sampling of the reception filter output at the optimum sampling time is realized by a multiplier. Then the threshold of the sampling result is decided, the optimal threshold is set to zero, the decision output result remains unchanged within one transmission symbol slot, and finally the recovery data with sampling rate of $1000 \mathrm{~Hz}$ is obtained by sampling at 10 times decay rate. Since the filtering delay of the transmitting filter and the receiving filter are designed to be 10 transmission symbol slots, the delay of 20 time slots in the transmission, plus the delay of the two time slots in the receiver sampling and decision recovery section, The received recovered data is delayed by 22 symbols compared with the transmission source data.

\section{Simulation results and analysis}

In the actual digital interconnection system, it is very difficult to completely eliminate inter-symbol interference, and the influence of inter-symbol crosstalk on the bit error rate can not find 
the statistic law which is easy to deal with in mathematics at present, and can not calculate accurately. In order to measure the performance of the baseband transmission system, in the laboratory, usually observe the waveform of the received signal with the oscilloscope to analyze the influence of the intersymbol interference and noise on the system performance, which is the eye diagram analysis method. In the ideal case of no intersymbol interference and noise, the waveforms are undistorted, each symbol will overlap, and eventually the "eye" and "eye" of the trace will be visible on the oscilloscope. When there is inter-symbol crosstalk, the waveform distortion, the symbol is not completely coincide, eye traces will be unclear, causing the "eye" part of the closure. If coupled with the effect of noise, the lines of the eye are blurred, and the "eye" is small. Therefore, the size of the "eye" indicates the degree of distortion, which reflects the strength of the intersymbol interference. It can be seen, the eye diagram can visually indicate the impact of intersymbol interference and noise, can evaluate the performance of a baseband transmission system advantages and disadvantages. It can also be used to adjust the characteristics of the receiver filter to reduce intersymbol interference and improve the transmission performance of the system. Figure 3 is the eye diagram when no noise, Figure 4 is the eye diagram after superimposed Gaussian noise.

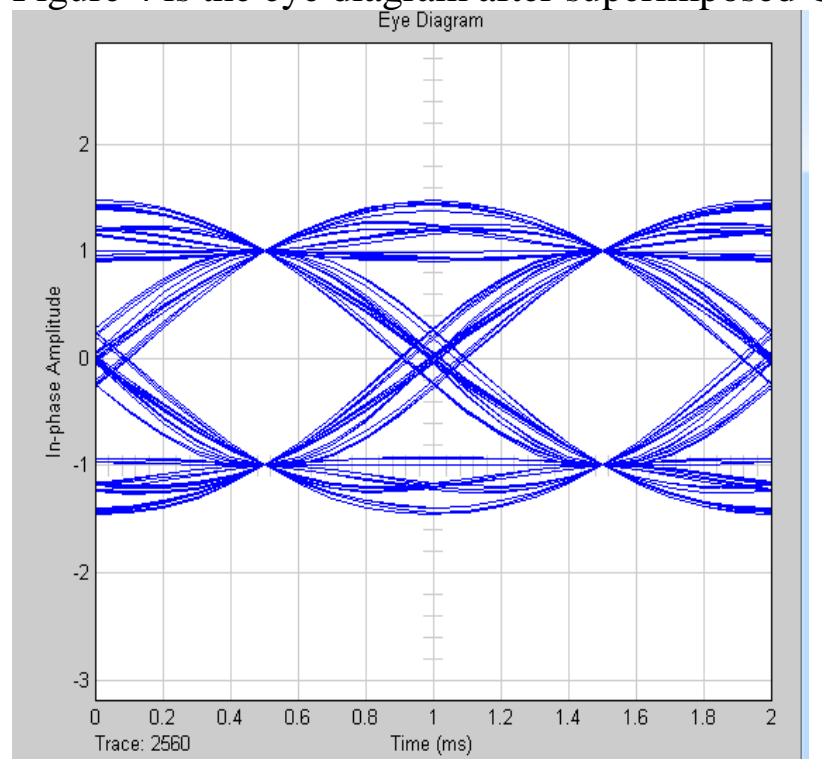

Fig.3 Eye Diagram When No Noise noise

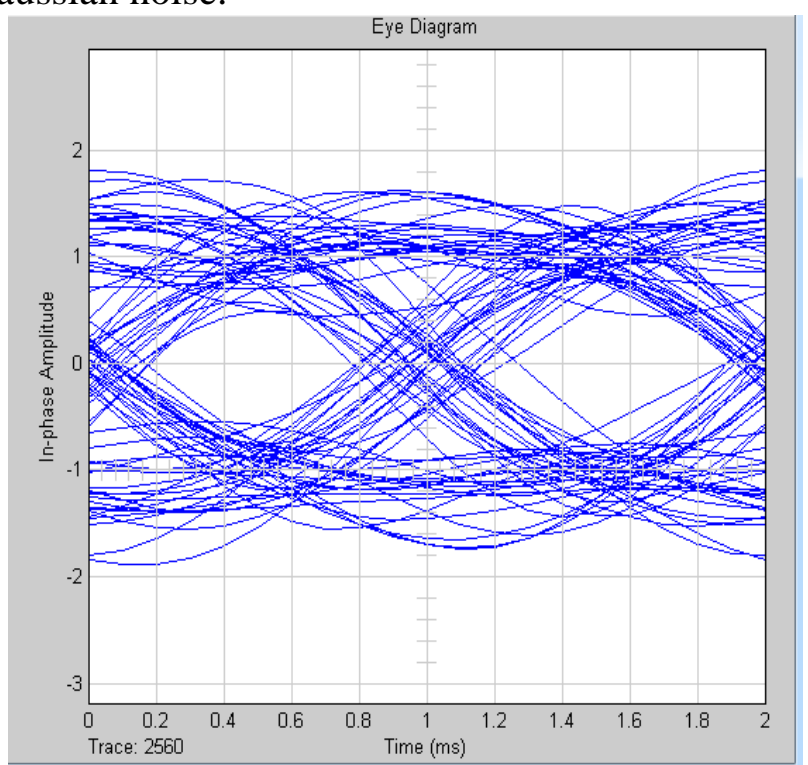

Fig.4 Eye Diagram after Superimposed Gaussian

Figure 5 shows the transmit and receive data waveforms. Run the model can be obtained from the monitor channel noise variance of 0.05 , the test bit error rate of 0.0068 .

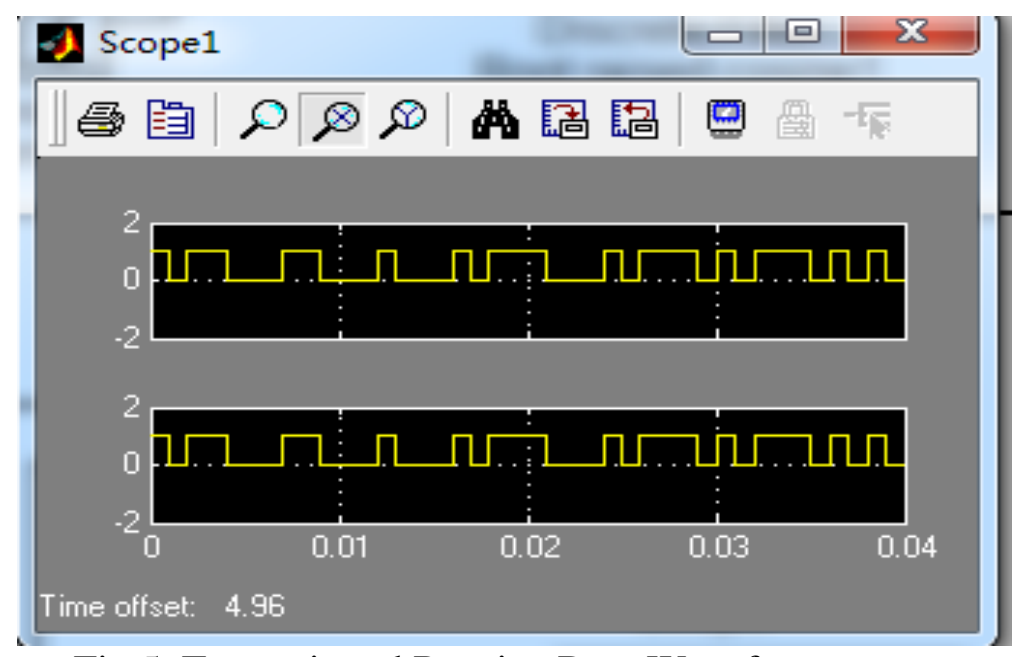

Fig.5 Transmit and Receive Data Waveforms 


\section{Conclusion}

The virtual simulation experiment is introduced into the experimental teaching of communication principle course, which can make up for the deficiency of hardware experiment and help to train the students' ability of design, analysis and innovation. In this paper, the digital baseband transmission system is simulated by using MATLAB software, and the possible factors of baseband signal transmission are fully considered. A binary bipolar digital baseband transmission system model is established. The system model can simulate Simulation and realization of digital baseband signal transmission function. The influence of channel SNR on system performance is analyzed by eye diagram and bit error rate (BER). It is shown that under the same condition, the larger the SNR is, the smaller the BER is, and the better the transmission quality. Analysis consistent. From the simulation results analysis and BER performance verification, bipolar digital baseband transmission system fully meet the design requirements.

\section{Acknowledgment}

This work was supported by the Hubei University of Science and Technology Teaching Research Project(2015-XB-007).

\section{References}

[1] Komine, Toshihiko, and Masao Nakagawa. "Fundamental analysis for visible-light communication system using LED lights." IEEE transactions on Consumer Electronics 50.1 (2004): 100-107.

[2] Heidari-Bateni, Ghobad, and Clare D. McGillem. "A chaotic direct-sequence spread-spectrum communication system." IEEE Transactions on communications 42.234 (1994): 1524-1527.

[3] Rappaport, Theodore S., et al. "Wideband millimeter-wave propagation measurements and channel models for future wireless communication system design." IEEE Transactions on Communications 63.9 (2015): 3029-3056.

[4] Stanger-Hall, Kathrin F., and James E. Lloyd. "Flash signal evolution in Photinus fireflies: character displacement and signal exploitation in a visual communication system." Evolution 69.3 (2015): 666-682.

[5] SLi, Jie, et al. "300-Gb/s eye-diagram measurement by optical sampling using fiber-based parametric amplification." IEEE Photonics Technology Letters13.9 (2001): 987-989.

[6] Breed, Gary. "Analyzing signals using the eye diagram." High Frequency Electronics 4.11 (2005): 50-53.

[7] Shake, Ippei, Hidehiko Takara, and Satoki Kawanishi. "Simple measurement of eye diagram and BER using high-speed asynchronous sampling." Journal of lightwave technology 22.5 (2004): 1296. 